\title{
JOURNAL OF THE INTERNATIONAL ARTHURIAN SOCIETY
}

\section{EDITOR-IN-CHIEF}

Leah Tether, Bristol

\section{DEPUTY EDITOR}

Samantha J. Rayner, UCL

\section{EDITORIAL BOARD}

Bart Besamusca, Utrecht

Frank Brandsma, Utrecht

Keith Busby, Madison, WI

Fabrizio Cigni, Rome

Cora Dietl, Gießen

Siân Echard, Vancouver

Christine Ferlampin-Acher, Rennes

Norris Lacy, University Park, PA

Carolyne Larrington, Oxford

Ceridwen Lloyd Morgan, Bangor

Andrew Lynch, Perth

Martine Meuwese, Utrecht

Matthias Meyer, Vienna

Ad Putter, Bristol

Raluca Radulescu, Bangor

José Carlos Ribeiro Miranda, Porto

Richard Trachsler, Zurich

Juan Miguel Zarandona, Valladolid

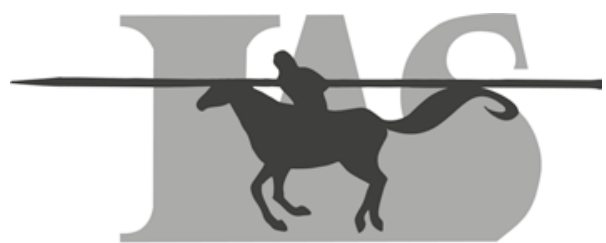


ABSTRACTED/INDEXED IN Baidu Scholar - CNKI Scholar (China National Knowledge Infrastructure) · CNPIEC · De Gruyter: IBR (International Bibliography of Reviews of Scholarly Literature in the Humanities and Social Sciences); IBZ (International Bibliography of Periodical Literature in the Humanities and Social Sciences) · EBSCO Discovery Service · ERIH PLUS (European Reference Index for the Humanities and Social Sciences) · Google Scholar · J-Gate · JournalTOCs · KESLINDSL (Korean National Discovery for Science Leaders) · Microsoft Academic $\cdot$ MLA International Bibliography · Naviga (Softweco) · Primo Central (ExLibris) · Publons · ReadCube · Summon (Serials Solutions/ProQuest) - TDNet · Ulrich's Periodicals Directory/ulrichsweb · WanFang Data · WorldCat (OCLC)

ISSN 2196-9353 · e-ISSN 2196-9361

All information regarding notes for contributors, subscriptions, Open access, back volumes and orders is available online at www.degruyter.com/jias

RESPONSIBLE EDITOR Dr. Leah Tether, University of Bristol, Department of English, 3-5 Woodland Road, Bristol, BS8 1TB, UK, Tel. +44 (0)1173 311027 , e-mail: leah.tether@bristol.ac.uk

JOURNAL MANAGER Claudia Hill, De Gruyter, Genthiner Straße 13, 10785 Berlin, Germany, Tel.: +49 (0)30 26005 - 172, Fax: +49 (0)30 26005 - 250, e-mail: claudia.hill@ degruyter.com

RESPONSIBLE FOR ADVERTISEMENTS Claudia Neumann, De Gruyter, Genthiner Straße 13, 10785 Berlin, Germany. Tel.: +49 (0)30.260 05-358, Fax: +49 (0) 30.260 05-226,

e-mail: anzeigen@degruyter.com

(C) 2018 Walter de Gruyter GmbH, Berlin/Munich/Boston

TYPESETTING fidus Publikations-Service, Nördlingen

PRINTING Franz X. Stückle Druck und Verlag e.K., Ettenheim

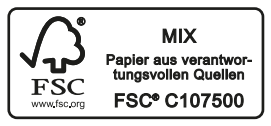

Offenlegung der Inhaber und Beteiligungsverhältnisse gem. § 7a Abs. 1 Ziff. 1, Abs. 2 Ziff. 3 des Berliner Pressegesetzes: Die Gesellschafter der Walter de Gruyter GmbH sind: Cram, Gisela, Rentnerin, Berlin; Cram, Elsbeth, Pensionärin, Rosengarten-Alvesen; Cram, Dr. Georg-Martin, Unternehmens-Systemberater, Stadtbergen; Cram, Maike, Wien (Österreich); Cram, Jens, Mannheim; Cram, Ingrid, Betriebsleiterin, Tuxpan/Michoacan (Mexiko); Cram, Sabina, Mexico, DF (Mexiko); Cram, Silke, Wissenschaftlerin, Mexico DF (Mexiko); Cram, Björn, Aachen; Cram, Berit, Hamm; Cram-Gomez, Susana, Mexico DF (Mexiko); Cram-Heydrich, Walter, Mexico DF (Mexico); CramHeydrich, Kurt, Angestellter, Mexico DF (Mexico); Duvenbeck, Birgitta, Oberstudienrätin i.R., Bad Homburg; Gädeke, Gudula, M.A., Atemtherapeutin/Lehrerin, Tübingen; Gädeke, Martin, Einzelunternehmer, Ingolstadt; Lubasch, Dr. Annette, Ärztin, Berlin; Schütz, Dr. Christa, Ärztin, Mannheim; Schütz, Sonja, Berlin; Schütz, Juliane, Berlin; Schütz, Antje, Berlin; Schütz, Valentin, Mannheim; Seils, Dorothee, Apothekerin, Stuttgart; Seils, Dr. Ernst-Albert, Pensionär, Reppenstedt; Seils, Gabriele, Dozentin, Berlin; Seils, Christoph, Journalist, Berlin; Siebert, John-Walter, Pfarrer, Oberstenfeld; Tran, Renate, Mediatorin, Zürich (Schweiz). 


\section{Contents}

Leah Tether and Samantha J. Rayner

Editorial -1

Mikayla Hunter

Memory, Gender and Recognition in Le Morte Darthur - 3

Kristina Hildebrand

The Other Cornwall Girl: Morgause in Twentieth-Century English

Literature -25

Michaela Wiesinger

Feenreste: Zur latenten Feenhaftigkeit hofferner Frauenfiguren in Diu Crône Heinrichs von dem Türlin — 46

Jean-Guy Gouttebroze

Melkin et les treize boules de cristal -70

Friedrich Michael Dimpel

des willn si bêde wârn bereit: Deviante Stimmen und wie Antikonie Handlungsautonomie postuliert -95

Matt Clancy

The Lost Tomb of Arthur and Guinevere at Glastonbury Abbey 123

$\mathbf{X X V I}^{\text {th }}$ International Arthurian Congress, Catania, July $2020-145$

Bi-annual JIAS Essay Prize Competition $2019 \_146$

\section{Obituary}

Philippe Ménard

Jacques Ribard (1926-2018) — 147 
